\title{
Intestinal microflora and immunoregulation
}

\author{
M Boirivant ${ }^{1}$, A Amendola $^{1}$ and A Butera ${ }^{1}$
}

Immune homeostasis at mucosal level results from controlled response to intestinal luminal antigens. Recent insights into the nature of inflammatory bowel diseases, derived mainly from studies of experimental models of colonic inflammation, strongly suggest that they can result from a loss of immune tolerance to antigens in the bacterial microflora. Investigations of the regulatory mechanisms operating at the mucosal level suggest that regulatory cells reactive to the intestinal microflora might play a role in cross-reactive protection toward different antigens. Expansion of microflora-reactive regulatory cells by probiotic administration is able to protect from experimental colitis. Characterization of regulatory cells in response to normal commensal flora, the basis of their development and the role of innate immunity in this process might contribute to the understanding of the development of inflammatory bowel diseases.

\section{INTRODUCTION}

Immune homeostasis at mucosal level results from controlled response to intestinal luminal antigens. ${ }^{1}$ In Crohn's disease, genetic and environmental factors interact to produce an immunopathogenic process that results in chronic relapsing intestinal inflammation. ${ }^{2}$ Recent insights into the nature of this disease, derived mainly from studies of experimental models of colonic inflammation, strongly suggest that it can result from a loss of immune tolerance to antigens in the bacterial microflora. This, in turn, can occur as a consequence of an absolute or relative defect in the function of regulatory $T$ cells or of an excessive immune response to these antigens that cannot be controlled by normal regulatory $\mathrm{T}$-cell fu nction. ${ }^{3}$

\section{REGULATORY MECHANISMS IN EXPERIMENTAL COLITIS}

In the last years we were involved in studies on experimental colitis pointing to the investigation of the regulatory mechanisms operating at the mucosal level and the requirements for their expansion. In these studies, we adopted as models of inflammation to be regulated, the inflammation associated with the intrarectal administration of 2,4,6-trinitrobenzene sulfonic acid (TNBS) or oxazolone, which induce a Th1and Th2-mediated inflammation, respectively. We started our observations by analyzing the relationship between IL-10 and TGF- $\beta$ in the protection from TNBS-colitis. We obtained such protection by feeding mice trinitrophenyl (TNP)-haptenated colonic proteins (a mixture of proteins obtained by homogenization of colonic specimens and then subjected to TNP-haptenization by TNBS) according to a protocol applied in an earlier study. ${ }^{4}$ As a result of such feeding, $\mathrm{T}$ cells producing TGF- $\beta$ and IL-10 appear in the lamina propria (LP) and are able to suppress TNBS-colitis in the same mouse or in a recipient mouse to which such cells are transferred. ${ }^{5}$ We then analyzed the relation between the two cytokines by selectively blocking the two cytokines during the induction of regulatory cytokines or during their expansion. We showed that, although anti-TGF- $\beta$ administration led to decreased TGF- $\beta$ production and loss of the regulatory effect, it had no effect on IL-10 production. In contrast, anti-IL-10 administration reduced both IL-10 and TGF- $\beta$ production. These latter findings suggested that the role of IL-10 in this context was to support the development of cells producing TGF- $\beta$ and that IL-10 itself exerted little regulatory effect. Finally, in cell transfer studies; we demonstrated that the effect of IL-10 does not occur during the initial induction of TGF- $\beta$-producing regulatory cells, as these cells may be also induced when IL-10 activity is blocked, but rather during the period of their expansion. Thus, these studies suggest that the function of IL-10 induced in the LP is to support the development of regulatory TGF- $\beta$-producing T cells, that are the proximal inhibitors of the colitis. ${ }^{5}$ In further studies, exploring the possibility to take an advantage of the lack of antigen-specificity in the suppressive effect of regulatory cytokine, we investigated the ability of regulatory cells induced by the oral administration of TNP-haptenated colonic proteins

${ }^{1}$ Department of Infectious, Parasitic and Immune-mediated Diseases, Immune-mediated Disease Section, Istituto Superiore di Sanità, Rome, Italy. Correspondence: M Boirivant (monica.boirivant@iss.it) 
to suppress a colitis induced by a different haptenating agent (oxazolone colitis). We found that the oral administration of TNP-haptenated colonic proteins, protected mice from the development of oxazolone colitis, albeit to a lesser extent than it protected mice from TNBS-colitis. ${ }^{6}$ It is important to note that the timing of the regulatory response with respect to the induction of inflammation is critical for its capacity to control (i.e., prevent) the inflammation, as a preexisting inflammatory response inhibits the effect of regulatory cells. This is probably because of the observed increase in both TNBS and oxazolone established colitis of SMAD7, an intracellular inhibitor of TGF- $\beta$ signaling. ${ }^{7}$ The cross-protection observed in this study, ${ }^{6}$ indeed arises from the fact that the regulatory cells are not antigenspecific in their suppressor function. However, this observation also suggests that some regulatory cells specific for luminal antigens are generated during the feeding, and that these cells are activated during the induction of colitis. The most likely possibility about specificity is that the fed TNP-haptenated colonic proteins lead to the induction of cells able to react not only with the inducing antigen originally administered to the mice but also with cross-reacting proteins in the mucosal microflora. Such proteins can act as activating antigens in the absence of the originally fed antigen.

\section{PROBIOTIC BACTERIA INTHE PREVENTION OF EXPERIMENTAL COLITIS}

The results obtained in this study, focused our attention on the microflora and on the possibility to expand regulatory cells by administration of high dose of probiotics that might act as activating antigens for microflora-reactive regulatory cells with prevention of a subsequent colitis.

We reasoned that an experimental setting consistent with our earlier observations should have been the administration of high dose of probiotics during a period of inflammatory quiescence. In initial studies, we showed that the oral administration of a mixture of probiotic bacteria (VSL\#3) to mice that have recovered from a first induction of TNBS-colitis, greatly reduces the severity of a subsequent second induction of TNBS-colitis. This protection was due to mononuclear cells in the LP of the treated mice, as it could be transferred to naive mice by adoptive transfer of these cells. Further studies showed that probiotic treatment was associated with the appearance of $\mathrm{CD} 4+$ regulatory $\mathrm{T}$ cells bearing cell-surface TGF- $\beta$ in its latent form (latency-associated peptide (LAP) + T cells). These cells proved to be protective cells, since LP cell populations from probiotic-treated mice depleted of these cells failed to transfer protection. Moreover, transferred protection was blocked by the administration of antiTGF- $\beta$ antibodies. Of considerable interest, the development of these cells was dependent on IL-10. In fact, the administration of probiotics was accompanied by the production of this cytokine and the treatment of mice with anti-IL-10R blocked the appearance of TGF- $\beta$-bearing regulatory cells. These studies provide evidence that probiotic effects are indeed linked to the ability to induce the development of IL-10-dependent TGF- $\beta$-bearing regulatory cells. ${ }^{8}$

In additional studies, we also investigated the effect of probiotic administration (VSL\#3) on CD4 + /CD25 + lymphocytes. We found a significant increase in the percentage of LP CD4+ cells expressing CD25 in mice treated with probiotics, when compared with untreated mice. This population minimally overlaps with the CD4 + /LAP + population, as in CD25gated cells LAP + cells represent the 25 and $33 \%$ of the LP CD $4+/ C D 25+$ cells in probiotic-treated and -untreated mice, respectively. In fact, the majority of CD25 + cells are indeed LAP - . Intriguing, this latter population has been reported to prevent colitis in the severe combined immunodeficiency a

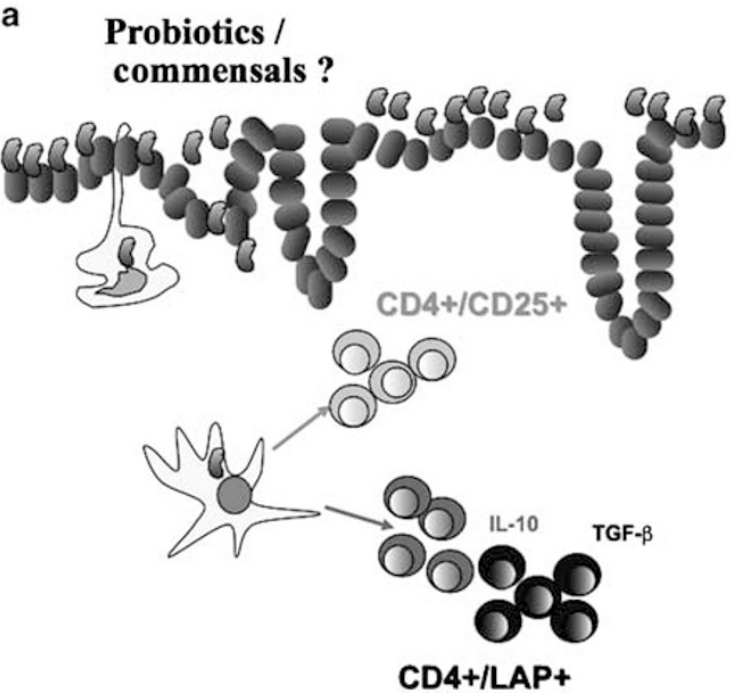

b
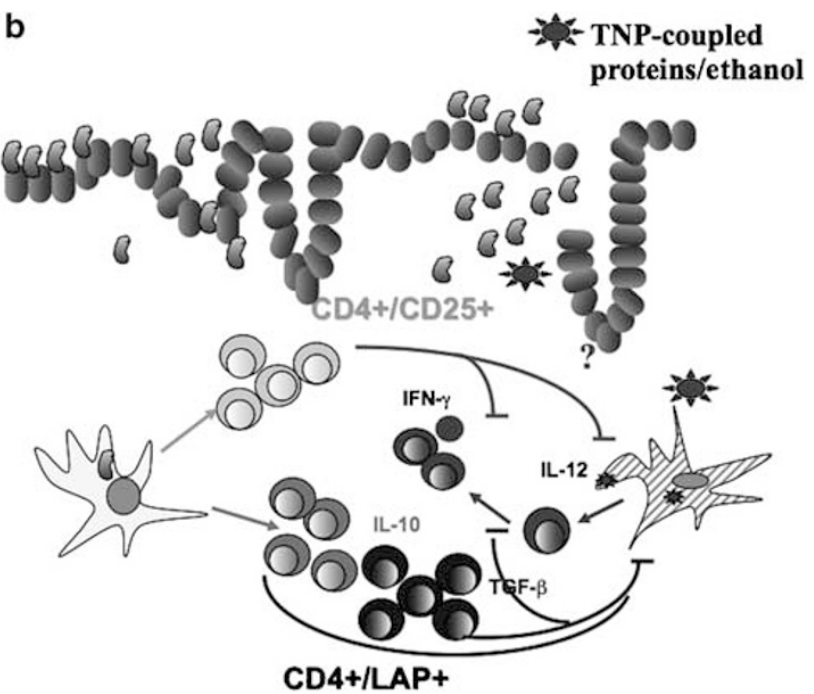

Figure 1 Effects of probiotic administration on LP regulatory cells. Probiotic administration induces the expansion of microorganism-reactive regulatory cells (a). These cells are activated by the microorganisms that penetrate the mucosa after the rupture of the epithelial barrier associated with the subsequent intrarectal administration of 2,4,6-trinitrobenzene sulfonic acid in ethanol $50 \%$ solution (b). Activated regulatory cells and their soluble factors are able to prevent or to rapidly inhibit the immune response to TNP-coupled proteins (b). 
disease transfer model of colitis, ${ }^{9}$ whereas it is unable to prevent TNBS-colitis, as suggested by the observation that LP cell populations from probiotic-treated mice depleted of LAP + cells (but not of CD25 + cells) failed to transfer protection from TNBS-colitis. Further work in which these regulatory cells are isolated and more thoroughly defined, as well as the knowledge of the requirements for their suppressive action, is needed to clarify this issue.

In a subsequent study, ${ }^{10}$ aimed to investigate ability of probiotics to modulate inflammation and the presence and type of mucosal regulatory cells, we conducted an open-label study, in which patients with ileal pouch-anal anastomosis for ulcerative colitis at different periods from surgery without signs and symptoms of pouchitis were randomized to VSL\#3 or no treatment for 12 months. Mucosal biopsies were collected at baseline and after 3,6, and 12 months. We observed that patients treated with VSL\#3, showed a significant reduction in the pouchitis disease activity index score associated with a significant reduction of tissue interleukin- $1 \beta$. They also showed a significant increase in the percentage of mucosal CD4 $+\mathrm{CD} 25$ high and CD $4+\mathrm{LAP}+$ cells compared with baseline values. Thus, LAP $+\mathrm{CD} 4+$ cells are also present in human LP and probiotic administration is able to induce in vivo expansion of mucosal regulatory cells in humans, confirming the data obtained in the murine system. ${ }^{8}$

\section{CONCLUSIONS}

The studies summarized above suggest that indeed intraluminal microbial antigens contribute to the expansion of LP regulatory cells (Figure 1). However, it remains to be defined whether the above outlined regulatory components, their interactions and requirements for expansion are also operating in response to normal commensal flora. The characterization of regulatory cells in response to normal commensal flora, the understanding of the basis of their development and the role of innate immunity in this process might contribute to the understanding of the pathogenesis of inflammatory bowel diseases.

\section{DISCLOSURE}

The authors have declared no financial interests.

C 2008 Society for Mucosal Immunology

\section{REFERENCES}

1. Strober, W., Fuss, I., Boirivant, M. \& Kitani, A. Insights into the mechanism of oral tolerance derived from the study of models of mucosal inflammation. Ann. NY Acad. Sci. 1029, 115-131 (2004).

2. Podolsky, D.K. Inflammatory bowel disease. N. Engl. J. Med. 347, 417-429 (2002).

3. Duchmann, R., Kaiser, I., Hermann, E., Mayet, W., Ewe, K. \& Meyer zum Buschenfelde, K.H. Tolerance exists towards resident intestinal flora but is broken in active inflammatory bowel disease (IBD). Clin. Exp. Immunol. 102, 448-455 (1995).

4. Neurath, M.F., Fuss, I., Kelsall, B.L. \& Strober, W. Experimental granulomatous colitis in mice is abrogated by induction of TGF-betamediated oral tolerance. J. Exp. Med. 183, 2605-2616 (1996).

5. Fuss, I.J., Boirivant, M., Lacy, B. \& Strober, W. The interrelated roles of TGF- $\beta$ and IL-10 in the regulation of experimental colitis. J. Immunol. 168, 900-908 (2002).

6. Boirivant, M., Strober, W. \& Fuss, I.J. Regulatory cells induced by feeding TNP-Haptenated colonic protein cross-protect mice from colitis induced by an unrelated hapten. Inflamm. Bowel Dis. 11, 48-55 (2005).

7. Boirivant, M. et al. Inhibition of Smad7 with a specific antisense oligonucleotide facilitates TGF-beta1-mediated suppression of colitis. Gastroenterology 131, 1786-1798 (2006).

8. Di Giacinto, C., Marinaro, M., Sanchez, M., Strober, W. \& Boirivant, M. Probiotics ameliorate recurrent Th1-mediated murine colitis by inducing IL-10 and IL-10-dependent TGF-\{beta\}-bearing regulatory cells. J. Immunol. 174, 3237-3246 (2005).

9. Oida, T. et al. CD4+CD25 - T cells that express latency-associated peptide on the surface suppress CD4+CD45Rbhigh-induced colitis by a TGF- $\beta$-dependent mechanism. J. Immunol. 170, 2516-2522 (2003).

10. Pronio, A. et al. Probiotic administration in patients with ileal pouch-anal anastomosis for ulcerative colitis is associated with expansion of mucosal regulatory cells. Inflamm. Bowel Dis. 14, 662-668 (2008). 